\title{
1 Pooled RNA: extraction free testing of saliva for SARS-CoV-2 detection
}

2 Orchid M. Allicock ${ }^{1 *}$, Devyn Yolda-Carr ${ }^{1 *}$, John A. Todd², Anne L. Wyllie ${ }^{1 \#}$

$3{ }^{1}$ Department of Epidemiology of Microbial Diseases, Yale School of Public Health, New Haven, CT 06510, 4 USA;

$5 \quad{ }^{2}$ Flambeau Diagnostics, Madison WI 53719, USA.

$7 \quad *$ These authors contributed equally to this article

$8 \quad$ \#Correspondence: anne.wyllie@yale.edu

10 Keywords: SARS-CoV-2; pooling; real-time RT-PCR; surveillance testing

\section{Abstract}

13 The key to limiting SARS-CoV-2 spread is to identify virus-infected individuals (both

14 symptomatic and asymptomatic) and isolate them from the general population. Hence, 15 routine weekly testing for SARS-CoV-2 in all asymptomatic (capturing both infected and non-infected) individuals is considered critical in situations where a large number of

17 individuals congregate such as schools, prisons, aged care facilities and industrial workplaces. Such testing is hampered by operational issues such as cost, test availability, access to healthcare workers and throughput. We developed the SalivaDirect RT-qPCR assay to increase access to SARS-CoV-2 testing via a low-cost, streamlined protocol 21 using self-collected saliva. To expand the single sample testing protocol, we explored multiple extraction-free pooled saliva testing workflows prior to testing with the 
23 SalivaDirect assay. A pool size of five, with or without heat inactivation at $65^{\circ} \mathrm{C}$ for 15

24 minutes prior to testing resulted in a positive agreement of $98 \%$ and $89 \%$, respectively,

25 and an increased Ct value shift of 1.37 and 1.99 as compared to individual testing of the

26 positive clinical saliva specimens. Applying this shift in $\mathrm{Ct}$ value to 316 individual,

27 sequentially collected, SARS-CoV-2 positive saliva specimen results reported from six

28 clinical laboratories using the original SalivaDirect assay, $100 \%$ of the samples would

29 have been detected ( $\mathrm{Ct}$ value $>45)$ had they been tested in the 1:5 pool strategy. The

30 availability of multiple pooled testing workflows for laboratories can increase test

31 turnaround time, permitting results in a more actionable time frame while minimizing

32 testing costs and changes to laboratory operational flow. 
medRxiv preprint doi: https://doi.org/10.1101/2022.01.16.22269390; this version posted January 17, 2022. The copyright holder for this preprint (which was not certified by peer review) is the author/funder, who has granted medRxiv a license to display the preprint in perpetuity.

It is made available under a CC-BY-NC-ND 4.0 International license .

\section{Introduction}

34 During the emergence and spread of the SARS-CoV-2 virus in 2020, the majority of 35 testing for the virus was aimed at diagnosing COVID-19 (the disease that it causes) in 36 patients presenting with symptoms characteristic of COVID-19. An infected person may develop COVID-19 disease symptoms 3 to 8 days post infection or may never develop symptoms (asymptomatic) (1-3). However, asymptomatic individuals can be infectious, carrying viral loads high enough to spread the virus to uninfected individuals. Soon thereafter it became clear that to control the spread of SARS-CoV-2 infection, a twopronged approach must be used in the general population to prevent viral spread from infected asymptomatic individuals to the non-infected population.

The first prong involves utilisation of physical barriers (e.g. face masks) to minimize virus spread via aerosols $(4,5)$. The second prong involves routine weekly testing for SARSCoV-2 in all asymptomatic (capturing both infected and non-infected) individuals at highrisk for infection $(6,7)$. Such testing is considered critical in situations where many individuals congregate such as schools, prisons, aged care facilities and industrial workplaces. Testing strategies rely on obtaining a respiratory tract specimen and an assay for the presence of SARS-CoV-2 antigen or genome.

It is generally considered that molecular tests for viral genome are more sensitive than the 51 antigen tests; however, they can be costly, can take days to return results and can be hard to scale for large population testing. Furthermore, for tests requiring a swab-based respiratory tract specimen, these can be uncomfortable and difficult to obtain, especially 54 under weekly self-collected specimen protocols, deterring individuals from participating in 55 testing (8). Early in the pandemic response, saliva emerged as an alternative specimen for 56 SARS-CoV-2 testing and by 2021 it became apparent that a self-collected specimen could 57 obviate the disadvantages of respiratory tract swab specimens. Importantly, the clinical 
medRxiv preprint doi: https://doi.org/10.1101/2022.01.16.22269390; this version posted January 17, 2022. The copyright holder for this preprint (which was not certified by peer review) is the author/funder, who has granted medRxiv a license to display the preprint in perpetuity.

It is made available under a CC-BY-NC-ND 4.0 International license .

sensitivities for SARS-CoV-2 detection were similar between respiratory tract swab and saliva specimens(9).

To address some of the limitations of testing, SalivaDirect ${ }^{\mathrm{TM}}$ was developed as an opensource protocol wherein clinical laboratories could adopt a streamlined, easy-to-use, inexpensive, scalable and flexible genomic (RT-qPCR) assay method for SARS-CoV-2 detection (10). Importantly the assay is based upon a simple self or observed saliva collection protocol. The SalivaDirect ${ }^{\mathrm{TM}}$ assay was developed to simplify testing individual saliva specimens; however, with the momentum around testing large-scale asymptomatic populations (e.g. school students, faculty and staff) where SARS-CoV-2 prevalence is low, in a cost-effective manner, a more scalable protocol is required for sustainable testing programs. We therefore investigated higher throughput protocols, wherein saliva specimens are pooled prior to testing with RT-qPCR. These pooled testing approaches were evaluated for the clinical sensitivity of SARS-CoV-2 detection.

\section{Methods}

\section{Ethics statement}

The use of de-identified saliva specimens from healthy or asymptomatic individuals was approved by the Institutional Review Board of the Yale Human Research Protection Program (Protocol ID. 2000028394) (11). Study participants were informed in writing about the purpose and procedure of the study, and consented to study participation through the act of providing the saliva sample; the requirement for written informed consent was waived by the Institutional Review Board. Additionally, the Institutional Review Board of the Yale Human Research Protection Program determined that the use of de-identified, remnant COVID-19-positive clinical samples obtained from laboratory partners for the RTqPCR testing conducted in this study is not research involving human subjects (Protocol ID: 2000028599). 
medRxiv preprint doi: https://doi.org/10.1101/2022.01.16.22269390; this version posted January 17, 2022. The copyright holder for this preprint (which was not certified by peer review) is the author/funder, who has granted medRxiv a license to display the preprint in perpetuity.

It is made available under a CC-BY-NC-ND 4.0 International license .

\section{Sample Collection}

All de-identified saliva samples used in the current study were collected unsupplemented into simple laboratory plastic tubes per the SalivaDirect protocol (12). All samples were tested with the SalivaDirect assay(13) in our research laboratory to confirm SARS-CoV-2 status. Samples were stored at $-80^{\circ} \mathrm{C}$ until further analysis.

\section{Pooled Sample Testing}

To understand the effect of sample dilution by pooling, on clinical sensitivity, a total of 20 saliva specimens which previously tested positive with the modified CDC assay RT-qPCR assay, with resulting cycle threshold $(\mathrm{Ct})$ values between 22.98 and 39.43 , were diluted $1: 5,1: 10$, and 1:20 with negative saliva specimens from healthcare workers(14). Undiluted specimens and pools were tested with the standard SalivaDirect RT-qPCR assay.

After identifying the optimal pool size, we performed an initial workflow evaluation using 5 different pooled samples, each composed of a single SARS-CoV-2-positive saliva sample pooled with 4 SARS-CoV-2-negative saliva samples, in a 1:5 dilution. To confirm our initial workflow findings and assess the sensitivity of viral detection when pooling, 20 additional pools ( 1 positive with 4 negative saliva specimens) were prepared and tested using the five different workflows.

The five different saliva pooling workflows investigated in both the initial and confirmation studies are depicted in Figure 1. All saliva samples were thawed on ice prior to testing and all samples were tested in duplicate. For workflows $A$ and $C$ (Figure 1a), $50 \mu$ of each sample, (including the SARS-CoV-2-positive saliva) were pooled to $250 \mu \mathrm{L}$ total volume, followed by vigorous vortexing. For workflow A, $50 \mu \mathrm{l}$ of the pooled sample was tested following the standard SalivaDirect protocol(10). For workflow $\mathrm{C}$ the remaining 
107 sample was treated with $10 \mu \mathrm{l}$ of proteinase $\mathrm{K}$ then heat inactivated before testing directly

108 without further treatment in the SalivaDirect RT-qPCR assay. Workflows B, D and E

109 (Figure 1b) involved incubating individual non-pooled samples at $65^{\circ} \mathrm{C}$ for 15 minutes

110 before combining $50 \mu \mathrm{l}$ of each sample into pools of 5 pre-treated samples. For workflow

$111 \mathrm{~B}, 50 \mu \mathrm{l}$ of the pool of pre-treated samples was tested through the standard SalivaDirect

112 protocol. For workflow E, $10 \mu \mathrm{l}$ of each of these pre-treated pools was removed and tested

113 with the SalivaDirect RT-qPCR assay without proteinase K treatment. Finally, for workflow

$114 \mathrm{D}, 10 \mu \mathrm{l}$ of proteinase $\mathrm{K}$ was added to the remaining volume of the pre-treated pool then

115 heat inactivated before testing in the SalivaDirect RT-qPCR assay.

117 Assessment of clinical Ct values with pooling

118 To evaluate the real-world potential loss of sensitivity on clinical samples with pooling, we

119 estimated the average change in $\mathrm{Ct}$ value for each pooled testing workflow using the

120 results from the 25-sample workflow confirmation study. We then estimated how the

121 change in Ct value would potentially impact assay sensitivity by applying the $\Delta \mathrm{Ct}$ value

122 (with pooling) to real world SalivaDirect RT-qPCR SARS-CoV-2 testing results. Six SARS-

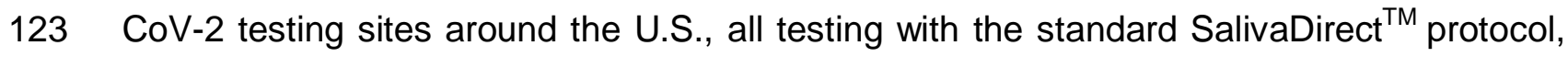

124 provided sequential testing results (Ct values for positive specimens) during August 2021

125 and to these values we applied the $\Delta$ Ct value that we estimated.

\section{Statistical analyses}

127 The correlation of Ct values between each workflow and the individual positive samples

128 was assessed using the Pearson correlation coefficient and represented graphically with

129 linear regression. The negative RT-PCR of the target gene was set at the Ct value of 45

130 for the statistical analysis. All statistical analyses were performed using GraphPad Prism 
medRxiv preprint doi: https://doi.org/10.1101/2022.01.16.22269390; this version posted January 17, 2022. The copyright holder for this preprint (which was not certified by peer review) is the author/funder, who has granted medRxiv a license to display the preprint in perpetuity. It is made available under a CC-BY-NC-ND 4.0 International license .

131 version 9 (GraphPad Software, San Diego, CA). For the calculation of percent positive 132 agreement, samples are considered positive at $\mathrm{Ct}<45$.

\section{Results}

\section{Pooling sizes and workflow selection}

135 We initially performed a limit of detection range-finding study to determine the impact of 136 sample dilution via pooling with the SalivaDirect RT-qPCR assay on detection sensitivities.

137 As pool size increased the resulting assay Ct values increased as well, generally in a 138 linear manner. The smallest change in Ct values (i.e. loss of assay sensitivity) of pooled 139 versus neat saliva was obtained with 1:5 pooling (1 positive and 4 negative saliva 140 samples). Thus a 1:5 pooling strategy was employed for workflow analysis. Our 141 preliminary results indicated that the SalivaDirect assay was able to detect SARS-CoV-2 142 in pooled saliva specimens with high virus loads, but additional testing was required to 143 optimize saliva pooling and processing workflows.

144 Table 1. Distribution of the Ct values of clinical saliva samples used for pooling.

\begin{tabular}{|c|c|c|}
\hline Ct range $^{*}$ & $\begin{array}{c}\text { No. samples Workflow A } \\
(\mathrm{n}=22)\end{array}$ & $\begin{array}{c}\text { No. samples Workflow B - E } \\
(\mathrm{n}=25)\end{array}$ \\
\hline $20.0-29.9$ & $7(32 \%)$ & $7(28 \%)$ \\
\hline $30.0-34.9$ & $5(23 \%)$ & $8(32 \%)$ \\
\hline $35.0-40.0^{* *}$ & $10(45 \%)$ & $10(40 \%)$ \\
\hline
\end{tabular}

145 *samples $<40 \mathrm{Ct}$ are considered positive for SARS-CoV-2

$146{ }^{* *}$ mean Ct for the CFX96 Touch RT-qPCR instrument when determining LOD for analytical sensitivity using 147 this set of reagents was 36.7 
medRxiv preprint doi: https://doi.org/10.1101/2022.01.16.22269390; this version posted January 17, 2022. The copyright holder for this preprint (which was not certified by peer review) is the author/funder, who has granted medRxiv a license to display the preprint in perpetuity.

It is made available under a CC-BY-NC-ND 4.0 International license .

148 Extrapolating from previous work (Watkins et al. 2021), we selected 5 workflows

149 representing different pooling strategies. Initial analyses using 5 pools showed that 4

150 workflows (A-D) provided similar results for most of the pools (see Table S1). Workflow E

151 provided a much larger shift in Ct values for all five pools (5.26) and hence loss of assay

152 sensitivity. As expected, a shift in Ct values (to higher) was noted for all five pooled

153 workflows compared the standard SalivaDirect RT-qPCR assay performed on the

154 undiluted positive sample. For workflows A-E, initial analysis of the differences in Ct

155 values between the pools and individual positive samples resulted in a Ct shift of 2.17 to

156 3.50. Workflow $E$ was omitted from further evaluations.

157 Workflow analysis

158 Twenty-five SARS-CoV-2 saliva specimens were used for pooling, with each pool

159 including one unique positive specimen and 4 negative specimens, to make 25 contrived

160 pools. The Ct values (obtained at the site of saliva collection) for SARS-CoV-2-positive

161 samples ranged from 22.98 to 39.43 . The majority (40 to $45 \%$ ) of the specimens had Ct

162 values $>35$ indicating a relatively low concentration of virus, 28 to $32 \%$ of the specimens

163 had Ct values <30 indicating a higher concentration of virus (Table 1, Table S2).

164 We assessed the sensitivity of each workflow by comparing to see which workflow had

165 the smallest shift in Ct values between the undiluted sample processed with SalivaDirect

166 RT-qPCR assay and the workflow in question, and by comparing which workflow had

167 the smallest number of pools dropping below the sensitivity threshold (between Ct 40 to

168 45). When compared to undiluted samples processed with the standard SalivaDirect

169 assay, Workflows $A$ and $B$ provided the highest sensitivity (Figure 2). Workflows $A$ and

170 B resulted in 3 and 1 pool(s) with Ct increases to 40 and 45, respectively. In contrast,

171 workflows $\mathrm{C}$ and $\mathrm{D}$ demonstrated the lowest in clinical sensitivity, with loss in detection

172 in $8 / 25$ and $10 / 25$ pools processed by these workflows respectively. 
173 Workflow A resulted in a positive agreement of $88.6 \%(86.4 \%$ and $91.0 \%$ for the

174 individual replicates), compared to the individual testing results using the standard

175 SalivaDirect protocol. Workflow B resulted in a 98\% positive agreement (100\% and $96 \%$

176 for the replicates), compared to the individual testing results using the standard

177 SalivaDirect protocol (Figure 2a). The positive agreement for workflows C and D were

178 less, with averages of $76 \%$ and $62 \%$, respectively.

179 A theoretical Ct shift of $\log _{2}(n)$ can be estimated for most RT-qPCR tests due to the 180 dilution of positive samples when pooled with negative samples. This means that for 181 pools of 5 , a Ct value shift of 2.3 would be expected. The $\mathrm{Ct}$ shift observed for 182 Workflows $A$ and $B$ were below this expected value, with Ct value shift of 1.99 and 1.37 183 respectively, confirming a slight loss of assay sensitivity. Workflows $\mathrm{C}$ demonstrated the 184 worst Ct value shift of 2.81 .

\section{Impact of pooling on clinical sensitivity}

186 To determine the pragmatic loss of clinical sensitivity with pooling before performing the

187 SalivaDirect RT-qPCR assay with workflows A-D, we queried six SalivaDirect CLIA 188 laboratories across the United States for the Ct values obtained from sequential testing of 189 saliva samples for SARS-CoV-2. These values and the breakdown of samples per site are 190 presented in Figure 3 (raw data available in Table S3). The average Ct value for the six 191 labs was 28.0. There was no statistical difference in Ct values across the labs. Out of a 192 total of 613 determinations across the labs, only 16 samples (2.6\%) had $\mathrm{Ct}$ values 193 between 38-40. Considering the calculated (from the confirmation study) worst case Ct 194 shift in pooling workflows A and B of 1.99 , and if all of the 613 determinations had been 195 made using workflow $\mathrm{A}$ or $\mathrm{B}$, these 16 samples would have shifted $\mathrm{Ct}$ to between 40 and 196 42. While considered negative using the individual testing workflow, these $2.6 \%$ of 197 samples would fall into a grey zone of 40-45 Ct values. 
medRxiv preprint doi: https://doi.org/10.1101/2022.01.16.22269390; this version posted January 17, 2022. The copyright holder for this preprint (which was not certified by peer review) is the author/funder, who has granted medRxiv a license to display the preprint in perpetuity.

It is made available under a CC-BY-NC-ND 4.0 International license .

\section{Discussion}

199 Widespread surveillance of asymptomatic individuals is one of the main methods of 200 controlling the spread of SARS-CoV-2. The pooling of samples before testing is a 201 resource-saving approach to increase testing capacity, especially for surveillance in a 202 population with a low infection rate (14), such as travellers, school populations and employees of large organisations. Additionally, testing these members of the community

204

205

206

207

208

209

210

211

212

213

214

215

216

217

218

219

serve as a proxy to the broader community, perhaps identifying larger outbreaks through family members and their associated activities. As saliva is easy to collect from a large number of people, pooling strategies are thus a natural extension to surveillance programs. While pooling saliva does impact assay sensitivity and potentially decrease virus detection, the actual impact appears to be minimal.

In the current study, we demonstrated that weakly positive samples (Ct values of 38 to 40 ) may be missed when testing pools of larger sizes (pools $>5$ ) when compared to testing samples individually. However, on the basis of the calculated relative sensitivity loss resulting from pooling, we looked at six datasets comprising 613 SARS-CoV-2 positive samples from across the U.S. Using such real world data we found that pooling saliva in groups of 5 samples prior to testing is expected to have minimal impact on clinical sensitivity. Based upon the lab reported Ct values only $2.6 \%$ of these samples would have shifted into a Ct 40-45 grey zone using the proposed SalivaDirect pooling workflows A or B. Importantly, if these samples were pooled, none would have become undetectable. It is advised that any sample pool resulting in Ct values between $40-45$ should be retested individually using the standard SalivaDirect protocol.

220 Surveillance programs for SARS-CoV-2 genomic testing in low prevalence populations 221 must be operationally pragmatic. First, they need to be cost-effective. Pooled testing 222 significantly reduces reagent costs, lab personnel cost, and lab resource needs. Second, 
medRxiv preprint doi: https://doi.org/10.1101/2022.01.16.22269390; this version posted January 17, 2022. The copyright holder for this preprint (which was not certified by peer review) is the author/funder, who has granted medRxiv a license to display the preprint in perpetuity.

It is made available under a CC-BY-NC-ND 4.0 International license .

223 these programs need to be easy to implement. Self-collection of a simple saliva specimen

224 obviates the need for healthcare workers to collect specimens and the associated

225 personal protective equipment. Third, programs should utilize existing resources for

226 sample collection. Self-collection of saliva can be performed anywhere and the resulting

227 specimen can be deposited at a drop site location (e.g. school or workplace entrance)

228 such that specimens from thousands of participants are collected in a parallel manner.

229 Pooling of specimens once received in the laboratory for testing should fit into established

230 laboratory accessioning and pre-analytic workflows. Finally, the end test results must

231 provide acceptable clinical sensitivities and specificities. We have shown that a saliva-

232 based RNA-extraction-free pooled (1:5) testing strategy results in detection of $97.4-100 \%$

233 SARS-CoV-2-positive samples, as compared to individual testing. Large pooled testing

234 programs have already demonstrated the efficacy of pooled saliva testing for helping to

235 keep schools safely open $(15,16)$, with pooled samples having a similar sensitivity to the

236 molecular testing of individual samples, in terms of both qualitative and quantitative

237 (comparable Ct values between pooled and individual samples) measures.

239 Throughout the pandemic, clinical laboratories have been hesitant to implement pooled 240 sample testing (17) due to: 1) stringent workflows which do not fit within existing laboratory

241 operations, 2) a lack of clear guidance on how to implement such methods and 3) the 242 perception that clinical sensitivity of the assay will be lost with pooling. The methods we 243 propose in the current study demonstrate minimal impact on assay sensitivity with 5 244 samples per pool, and are straightforward extensions of a simple SARS-CoV-2 testing 245 method which can be easily conducted manually, without requiring additional investment. 246 SalivaDirect is a flexible extraction-free platform for RT-qPCR testing. For ease of 247 implementation and safety of lab personnel, multiple workflows (18) were developed for 
248 the testing of individual samples. We sought to extend this level of flexibility for labs

249 seeking to offer pooled testing. We demonstrated that workflows A and B provide the best

250 assay sensitivity, with B providing a heat pre-treatment step for labs who require it by local

251 Environmental Health and Safety guidelines. Consequently, workflows A and B were

252 selected as the proposed approaches for pooling of the SalivaDirect test.

253 Overall, surveillance testing is not generally easy, requiring a pivot by traditionally clinical

254 diagnostic labs, especially when scalable protocols do not exist. Thus, when a decrease in

255 positive cases are observed, there is a psychological and practical desire to decrease

256 testing. However, these dips in COVID-19 cases can lead to a decrease in pandemic 257 preventative measures, which inevitably leads to disease resurgence. Additionally, with 258 the introduction of different variants of concern, the need for affordable and sustainable 259 mass testing strategies only becomes more urgent. Our findings suggest that combining 260 saliva with a practical pooling protocol will enable easier SARS-CoV-2 surveillance testing, 261 especially in resource-limited settings. Such pooled testing has the potential to 262 significantly reduce the overall number of tests and associated costs. This would in turn 263 operationally permit an increased frequency of testing, meaning an increased likelihood of 264 detecting individuals earlier in their infection. This approach should allow broader 265 screening in schools and workplaces for SARS-CoV-2 testing and importantly lay the 266 foundation for managing future upper respiratory infection mediated pandemics.

\section{Data Availability}

Data from this study is available in the supplemental information. 
272 A.L.W. conceived the study and developed the study protocol. D.A.Y.C. executed the

273 study. A.L.W. co-ordinated external laboratory data. O.M.A. and A.L.W. analyzed the data.

274 O.M.A. assisted with the design of the statistical analysis. J.A.T., D.A.Y-C, O.M.A., and

275 A.L.W. wrote and edited the manuscript.

276

277 Acknowledgements

278 We thank our external laboratory partners for their time and cooperation to make this

279 valuable extension of the SalivaDirect assay possible for other laboratories around the 280 country. This work was funded by Flambeau Dx (A.L.W) and Fast Grant from Emergent 281 Ventures at the Mercatus Center at George Mason University (A.L.W).

283 Figures

284 Figure 1. The SalivaDirect ${ }^{\mathrm{TM}}$ pooled testing workflows evaluated in the study. (A) For 285 workflows $\mathrm{A}$ (purple) and $\mathrm{C}$ (gold), $50 \mu$ l of SARS-CoV-2-positive saliva and $200 \mu \mathrm{L}$ total 286 volume of four SARS-CoV-2-negative saliva samples (50 $\mu$ l each) were pooled and 287 vigorously vortexed to mix. For workflow A, 50 $\mu$ l of the pooled sample was tested 288 following the standard SalivaDirect protocol. For workflow $\mathrm{C}$ the remaining sample was 289 treated with $10 \mu \mathrm{l}$ of proteinase $\mathrm{K}$ then heat inactivated before testing directly without 290 further treatment in the SalivaDirect ${ }^{\mathrm{TM}}$ RT-qPCR assay. (B) For workflows $\mathrm{B}, \mathrm{D}$ and $\mathrm{E}$, 291 individual, non-pooled samples were incubated at $65^{\circ} \mathrm{C}$ for 15 minutes before combining $29250 \mu \mathrm{l}$ of each sample into pools of 5 pre-treated samples. For workflow B (pink), $50 \mu \mathrm{l}$ of 293 the pool of pretreated samples was tested through the standard SalivaDirect protocol. For 294 workflow $\mathrm{E}$ (orange), $10 \mu \mathrm{l}$ of each of these pre-treated pools was removed and tested 
295 with the SalivaDirect ${ }^{\mathrm{TM}}$ RT-qPCR assay without proteinase $\mathrm{K}$ treatment. Finally, for

296 workflow D (blue), $10 \mu \mathrm{l}$ of proteinase $\mathrm{K}$ was added to the remaining volume of the pre-

297 treated pool then heat inactivated before testing in the SalivaDirect ${ }^{\mathrm{TM}} \mathrm{RT}$-qPCR assay.

299 Figure 2. SARS-CoV-2 N1 gene detection with individual saliva-based RNAextraction-free RT-qPCR testing versus pooled sample testing using Workflows A-

D. A) N1 detection of one positive sample pooled and tested with equal volumes of 4 negative samples correlated to the $\mathrm{Ct}$ value obtained when samples were tested 303 individually. B). Ct values obtained from each sample tested individually and when 304 combined with 4 negative samples and tested with each of the workflows. The N1 Ct cutoff 305 for classifying individual samples as positive is 40 (as indicated by the grey area under the 306 horizontal dashed line. No cutoff was set for the pooled samples. B) line - median values

Figure 3 Detection of SARS-CoV-2 N1 gene persists when Ct value shift from 


\section{References}

315 1. Wells PM, Doores KJ, Couvreur S, Nunez RM, Seow J, Graham C, Acors S, Kouphou N, Neil SJD, Tedder RS, Matos PM, Poulton K, Lista MJ, Dickenson RE, Sertkaya H, Maguire TJA, Scourfield EJ, Bowyer RCE, Hart D, O'Byrne A, Steel KJA, Hemmings O, Rosadas C, McClure MO, Capedevilla-Pujol J, Wolf J, Ourselin S, Brown MA, Malim MH, Spector T, Steves CJ. 2020. Estimates of the rate of infection and asymptomatic COVID-19 disease in a population sample from SE England. J Infect 81:931-936.

2. Bai Y, Yao L, Wei T, Tian F, Jin D-Y, Chen L, Wang M. 2020. Presumed Asymptomatic Carrier Transmission of COVID-19. JAMA 323:1406-1407.

3. Li R, Pei S, Chen B, Song Y, Zhang T, Yang W, Shaman J. 2020. Substantial undocumented infection facilitates the rapid dissemination of novel coronavirus (SARS-CoV-2). Science 368:489-493.

4. Asadi S, Cappa CD, Barreda S, Wexler AS, Bouvier NM, Ristenpart WD. 2020. Efficacy of masks and face coverings in controlling outward aerosol particle emission from expiratory activities. Sci Rep 10:15665.

5. Leung NHL, Chu DKW, Shiu EYC, Chan K-H, McDevitt JJ, Hau BJP, Yen H-L, Li Y, Ip DKM, Peiris JSM, Seto W-H, Leung GM, Milton DK, Cowling BJ. 2020. Respiratory virus shedding in exhaled breath and efficacy of face masks. Nat Med 26:676-680.

6. Hellewell J, Russell TW, Investigators S, Field Study T, Crick C-C, group CC-w, 
effectiveness of routine asymptomatic PCR testing at different frequencies for the detection of SARS-CoV-2 infections. BMC Med 19:106.

7. Paltiel AD, Zheng A, Walensky RP. 2020. Assessment of SARS-CoV-2 Screening Strategies to Permit the Safe Reopening of College Campuses in the United States. JAMA Netw Open 3:e2016818.

8. Nacher M, Mergeay-Fabre M, Blanchet D, Benois O, Pozl T, Mesphoule P, Sainte-

Biche B, Faurous W, Chaizemartin L, Fahrasmane A, Rochemont D, Diop F, Niang M, Pujo J, Vignier N, Dotou D, Vabret A, Demar M. 2021. Diagnostic accuracy and acceptability of molecular diagnosis of COVID-19 on saliva samples relative to nasopharyngeal swabs in tropical hospital and extra-hospital contexts: The

9. Tan SH, Allicock O, Armstrong-Hough M, Wyllie AL. 2021. Saliva as a goldstandard sample for SARS-CoV-2 detection. Lancet Respir Med doi:10.1016/s2213-2600(21)00178-8.

10. Vogels CBF, Watkins AE, Harden CA, Brackney DE, Shafer J, Wang J, Caraballo C, Kalinich CC, Ott IM, Fauver JR, Kudo E, Lu P, Venkataraman A, Tokuyama M, Moore AJ, Muenker MC, Casanovas-Massana A, Fournier J, Bermejo S, Campbell M, Datta R, Nelson A, Yale IRT, Dela Cruz CS, Ko AI, Iwasaki A, Krumholz HM, Matheus JD, Hui P, Liu C, Farhadian SF, Sikka R, Wyllie AL, Grubaugh ND. 2021.

SalivaDirect: A simplified and flexible platform to enhance SARS-CoV-2 testing capacity. Med (N Y) 2:263-280.e6.

11. Allicock OM, Petrone ME, Yolda-Carr D, Breban M, Walsh H, Watkins AE, 
collection devices for doi:10.1101/2021.02.01.21250946.

362 363

364 365

366

367

368

369

370

371

372

373

374

375

376

377

378

379

380

381

382

12. FDA. 2020. SalivaDirect - EUA Summary. https://www.fda.gov/media/141192/. Accessed 12/21/2021.

13. Vogels C, Orchid M, E D, Chaney C, Isabel M, Grubaugh N, Anne L. SalivaDirect ${ }^{\mathrm{TM}}$ : $\quad$ RNA extraction-free $\quad$ SARS-CoV-2 diagnostics v6 (protocols.io.btdnni5e). protocolsio doi:10.17504/protocols.io.btdnni5e.

14. Watkins AE, Fenichel EP, Weinberger DM, Vogels CBF, Brackney DE, CasanovasMassana A, Campbell M, Fournier J, Bermejo S, Datta R, Dela Cruz CS, Farhadian SF, Iwasaki A, Ko Al, Grubaugh ND, Wyllie AL. 2021. Increased SARS-CoV-2 Testing Capacity with Pooled Saliva Samples. Emerg Infect Dis 27:2-5.

15. Mendoza RP, Bi C, Cheng H-T, Gabutan E, Pagaspas GJ, Khan N, Hoxie H, Hanna S, Holmes K, Gao N, Lewis R, Wang H, Neumann D, Chan A, Takizawa M, Lowe J, Chen X, Kelly B, Asif A, Barnes K, Khan N, May B, Chowdhury T, Pollonini G, Gouda N, Guy C, Gordon C, Ayoluwa N, Colon E, Miller-Medzon N, Jones S, Hossain R, Dodson A, Weng M, McGaskey M, Vasileva A, Lincoln AE, Sikka R, Wyllie AL, Berke EM, Libien J, Pincus M, Premsrirut PK. 2021. Implementation of a pooled surveillance testing program for asymptomatic SARS-CoV-2 infections in K12 schools and universities. EClinicalMedicine 38:101028.

16. Vander Schaaf NA, Fund AJ, Munnich BV, Zastrow AL, Fund EE, Senti TL, Lynn AF, Kane JJ, Love JL, Long GJ, Troendle NJ, Sharda DR. 2021. Routine, CostEffective SARS-CoV-2 Surveillance Testing Using Pooled Saliva Limits Viral Spread on a Residential College Campus. Microbiol Spectr 9:e0108921. 
medRxiv preprint doi: https://doi.org/10.1101/2022.01.16.22269390; this version posted January 17, 2022. The copyright holder for this preprint

(which was not certified by peer review) is the author/funder, who has granted medRxiv a license to display the preprint in perpetuity. It is made available under a CC-BY-NC-ND 4.0 International license.

383 17. Fenichel EP, Tobias Koch R, Gilbert A, Gonsalves G, Wyllie AL. 2021. $384 \quad$ Understanding the Barriers to Pooled SARS-CoV-2 Testing in the United States. 385 Microbiology Spectrum 9.

386

387

388

389

390
18. Allicock OM, Yolda-Carr D, Earnest R, Breban M, Vega N, Ott IM, Kalinich C, Alpert T, Petrone M, Wyllie AL. 2021. Method versatility in RNA extraction-free PCR detection of SARS-CoV-2 in saliva samples. medRxiv doi:10.1101/2021.12.27.21268334. 
A
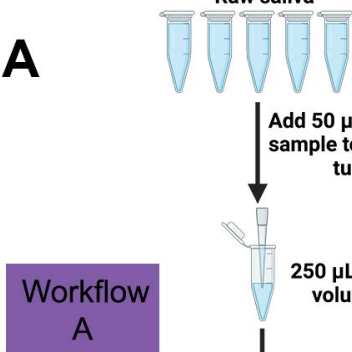

Take $\mathbf{5 0} \boldsymbol{\mu L}$ for testing by standard SalivaDirect Protocol SalivaDirect Protoco

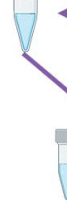

Add $2.5 \mu \mathrm{L}$ of proteinase $\mathrm{K}$

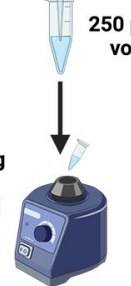

Add $50 \mu \mathrm{L}$ of each sample to a single tube

$250 \mu \mathrm{L}$ final volume

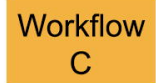

Add $10 \mu \mathrm{L}$ of proteinase $k$ to the pool

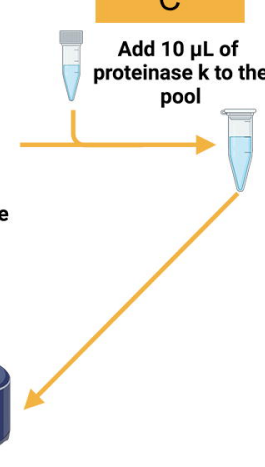

Mix to combine

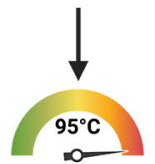

Inactivate proteinase $k$ $\downarrow 5 \mu \mathrm{L}$

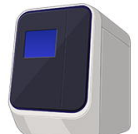

Duplex RT-qPCR Targets N1+ RP

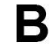

Raw saliva

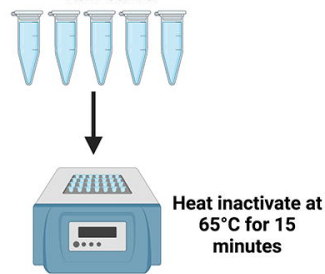

Add $50 \mu \mathrm{L}$ of each sample to a single tube

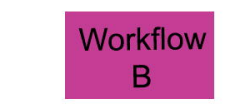

by standard SalivaDirect protocol
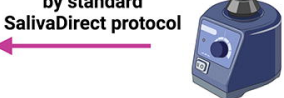

$250 \mu \mathrm{L}$ final volume

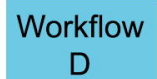

$\downarrow$

Add $10 \mu \mathrm{L}$ of proteinase $k$ to the pool

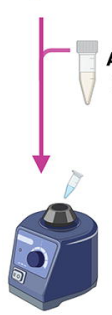

Add $2.5 \mu \mathrm{L}$ of proteinase $k$

Mix to combine
Mix to combine<smiles>C=CC=C</smiles>

$95^{\circ} \mathrm{C}$

proteinase $k$

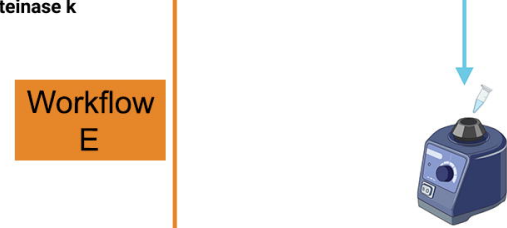

Mix to combine

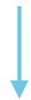

$5 \mu \mathrm{L}$

$95^{\circ} \mathrm{C}$

nactivate proteinase $\mathbf{k}$ 
A Workflow A
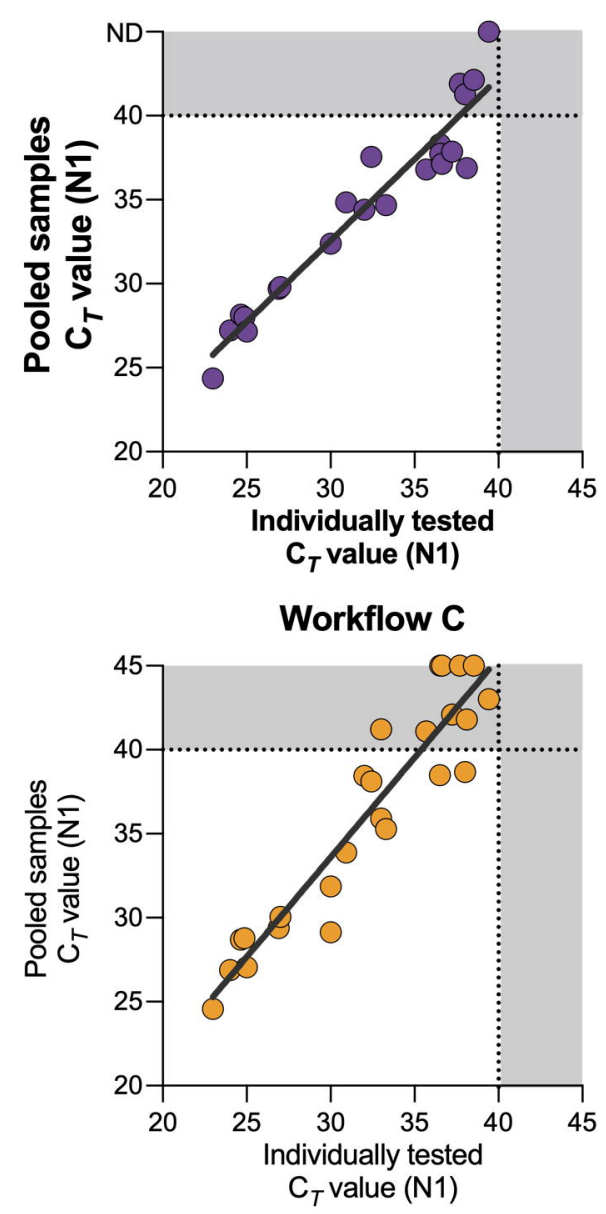

Workflow B

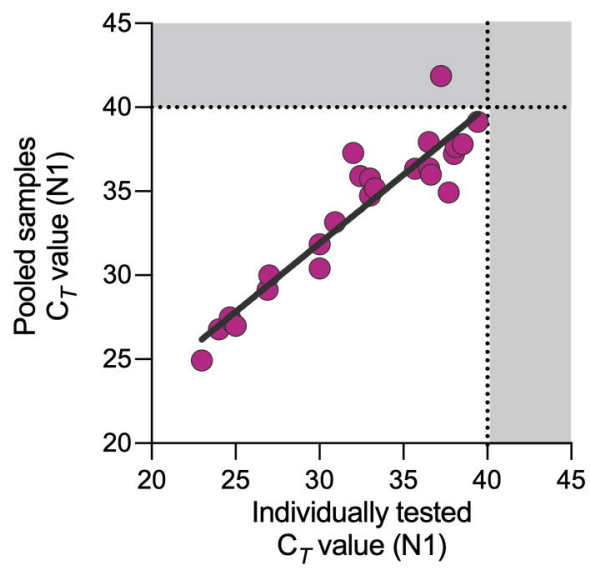

Workflow D

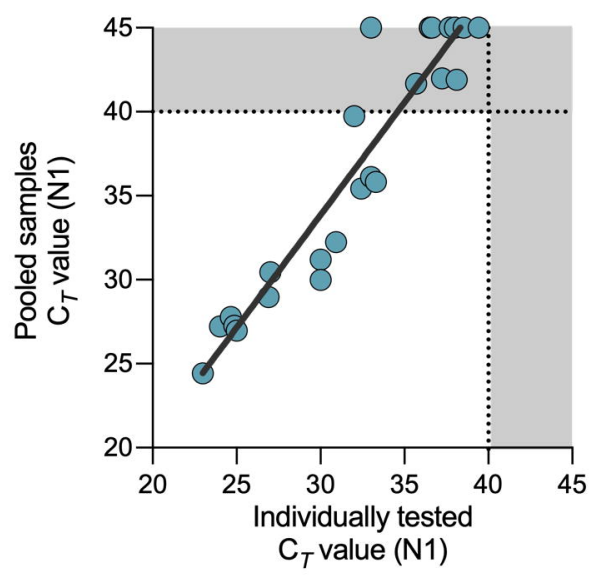

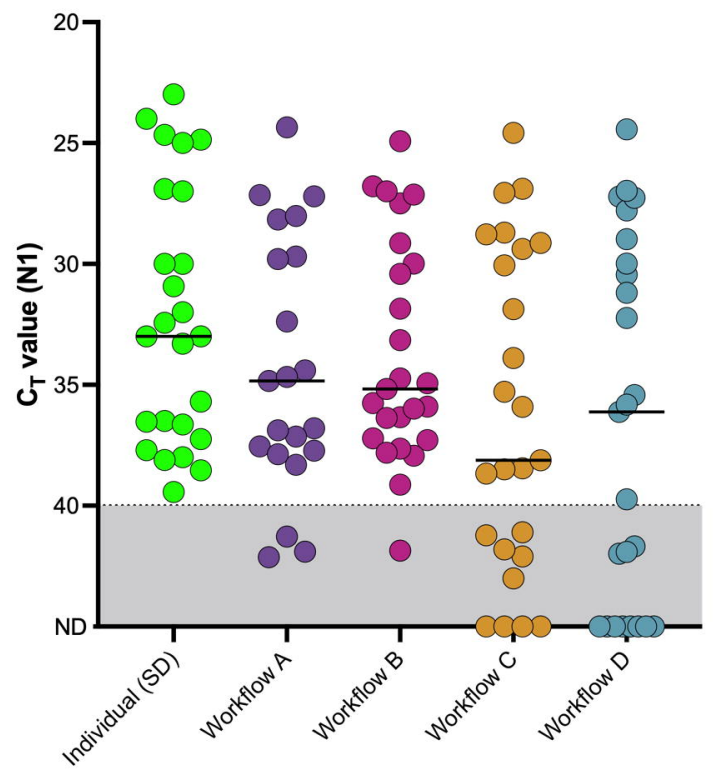






